\title{
Methods for constructing Collatz numbers
}

\author{
Abdullah N. Arslan \\ Department of Computer Science, Texas A\&M University-Commerce \\ Commerce, TX 75428, USA \\ e-mail: abdullah.arslanetamuc.edu
}

Received: 7 April 2016

Accepted: 7 May 2018

\begin{abstract}
The Collatz conjecture is among the unsolved problems in mathematics. It says that if we take any natural number $x$; divide it by two if $x$ is even, and multiply it by 3 and add 1 if $x$ is odd; and repeat this rule on the resulting numbers, eventually we obtain 1 . For a given positive integer $x$, we say that $x$ is a Collatz number if the claim of the conjecture is true for $x$. Computer verification reveals a large range of Collatz numbers. We develop methods by which we construct sets of Collatz numbers.
\end{abstract}

Keywords: Collatz conjecture, Collatz sequence.

2010 Mathematics Subject Classification: 11B83, 11Y55, 11A99, 11 B99.

\section{Introduction}

Lothar Collatz conjectured that if we take any natural number $x$ and do the following: if $x$ is even, divide it by 2 (divide by 2 rule); if $x$ is odd, multiply it by 3 and add 1 ( $3 x+1$-rule); if we repeat these steps indefinitely on the resulting numbers then we will eventually obtain 1 . Proving or disproving this conjecture has been among the unsolved problems in mathematics since it was stated by Collatz in 1937. It has also been among the problems whose solution will be awarded by a cash prize [7]. Paul Erdös made the following strong comment on this conjecture: "Mathematics may not be ready for such problems." [6]. The problem has also been known under other names $[2,3,4,5]$.

We consider sequences of integers generated by the rules described in the Collatz conjecture. For any given positive integer $x$ we call each such sequence starting with $x$ a Collatz sequence for $x$. Let $f_{i}(x)$ denote the number in a Collatz sequence for $x$ at step $i$ starting with initial number $x$ 
at step 0 . We can define $f_{i}(x)$ as the following function: for every positive integer $x, f_{0}(x)=x$, and for $i>0$,

$$
f_{i}(x)= \begin{cases}3 f_{i-1}(x)+1, & \text { if } f_{i-1}(x) \text { is odd } \\ f_{i-1}(x) / 2, & \text { otherwise, i.e. if } f_{i-1}(x) \text { is even }\end{cases}
$$

The Collatz conjecture claims that for any value of positive integer $x$, there exists step $i(i \geq 0)$ such that $f_{i}(x)=1$. Note that once 1 is obtained in the sequence then continuing the steps is not interesting because the subsequent integers will be 4,2 , and then 1 , and this pattern will repeat forever. Therefore, if 1 is obtained, we are not interested in the rest of the generated integers in the sequence. A positive integer $x$ has a 1-yielding Collatz sequence $f_{0}(x) f_{1}(x) \ldots f_{i}(x)$ if $f_{i}(x)=1$ at some step $i$. If $f_{i}(x)=1$ exists, the smallest $i$ is considered. The shortest 1 -yielding Collatz sequence for $x$ is the shortest sequence $f_{0}(x) f_{1}(x) \ldots f_{i}(x)$ where $f_{i}(x)=1$. The operation at step $i-1$ in generating $f_{i}$ from $f_{i-1}$ depends on the parity of $f_{i-1}$. Let 0,1 indicate respectively the divide by 2 , and the $3 x+1$ rule (operation). For a given Collatz sequence starting with $x$, we call the corresponding binary sequence of operations the Collatz operation sequence for $x$. There exists a Collatz operation sequence for $x$ that yields 1 at some step $i$ if $x$ has a 1 -yielding Collatz sequence. For $x$, the shortest 1-yielding Collatz operation sequence is the Collatz operation sequence that corresponds to the shortest 1-yielding Collatz sequence for $x$. We say that a positive integer $x$ is a Collatz number if $x$ has a 1-yielding Collatz sequence.

Let $\Theta(x)$ be the number of Collatz numbers not exceeding $x$. Krasikov [8] showed that $\Theta(x)>c x^{3 / 7}$ for some positive constant $c$. Andrei and Masalagiu [1] showed that $\frac{2^{m+1}\left(2^{3^{m} n}+1\right)}{3^{m+1}}$ is a Collatz number for all natural numbers $m, n$, where $n \in\{6 t+1,6 t+5 \mid$ for some natural number $t\}$. This is the most relevant result in the literature for our work as we also report new Collatz numbers in the current paper.

We do not attempt to prove or disprove the Collatz conjecture in this paper. We develop methods by which we can construct new Collatz numbers. By this kind of efforts, we can gain more insight into the conjecture, and discover some building blocks for forming larger sets of Collatz numbers. Clearly, the union of these sets is also a set of Collatz numbers. Our construction methods imply that for any given positive integer $B$, there exist odd Collatz numbers larger than $B$ with Collatz operation sequences in at least two different forms.

We explain our notation in Section 2. In Section 3 we show two methods to construct new positive odd Collatz numbers from the set of computer-verified Collatz numbers. Further, in Section 4, we construct two infinite sets of odd Collatz numbers. We summarize the results in Section 5 .

\section{Notation}

For any nonnegative integer $K$, we denote by $0^{K}$ a sequence of $K$ zeros, and by $\{0,1\}^{K}$ any $K$-bit sequence. Both $0^{0}$ and $\{0,1\}^{0}$ give the null sequence. For a given bit sequence $x,(x)_{2}$ denotes the corresponding binary number. For two bit-sequences $x$ and $y, x y$ denotes the binary number $(x y)_{2}$ formed by concatenating bit sequences $x$ and $y$ ( $x$ in the high order bits and $y$ in 
the low order bits). If a binary number $(x)_{2}$ yields a binary number $(y)_{2}$ in zero or more steps using the rules defined in Eq. (1) then we state this by the following expression: $x \Rightarrow^{*} y$, or equivalently, $(x)_{2} \Rightarrow^{*}(y)_{2}$. We also use them in a mixed way, e.g. $x \Rightarrow^{*}(y)_{2}$. When the derivation takes exactly one step then we use $\Rightarrow$ instead of $\Rightarrow *$.

Let $C$ denote the largest integer such that the Collatz conjecture has been verified by computer for all starting values in $[1, C]$. Currently $C=5 \times 2^{60}$ [9]. We use this value of $C$ in the rest of the paper.

\section{New finite sets of odd Collatz numbers}

Clearly, for every non-negative $n, 2^{n}$ has a 1-yielding Collatz sequence of length $n+1$ which contains all powers of 2 from power $n$ to power 0 .

Consider $\left(x 0^{n}\right)_{2}$ for a non-negative integer $n,\left(x 0^{n}\right)_{2}=(x)_{2} 2^{n} \Rightarrow(x)_{2} 2^{n-1} \Rightarrow{ }^{*}(x)_{2}$ after a sequence of $n$ divide-by-2 operations. If $(x)_{2} \leq C$ then the integer $\left(x 0^{n}\right)_{2}$ has a 1-yielding Collatz sequence since all integers up to $C$ have been verified by computer as Collatz numbers. In other words, if $(x)_{2} \leq C,\left(x 0^{n}\right)_{2}$ is a Collatz number for all non-negative integers $n$. In the rest of the paper, we focus on odd Collatz numbers.

Lemma 1. For every positive integer $K$ that satisfies $3^{\lceil K / 2\rceil}+2 \leq C$, the integer $2^{K}+1$ is an odd Collatz number.

Proof. For all $K \geq 1,2^{K}+1$ is a positive odd integer for which a Collatz sequence is described in Table 1. After step 0, steps are grouped by three as shown in the table.

\begin{tabular}{|l|l|c|l|l|}
\hline step $j$ & operation & group & $\begin{array}{l}\text { result } f_{j} \\
\text { (current integer in the sequence) })\end{array}$ & parity \\
\hline 0 & initialize & & $2^{K}+1$ & odd \\
1 & $3 x+1$ rule & 1 & $3\left(2^{K}\right)+4$ & even \\
2 & divide by 2 & 1 & $3\left(2^{K-1}\right)+2$ & even \\
3 & divide by 2 & 1 & $3\left(2^{K-2}\right)+1$ & edd \\
$3.1+1$ & $3 x+1$ rule & 2 & $3^{2}\left(2^{K-2}\right)+4$ & even \\
$3.1+2$ & divide by 2 & 2 & $3^{2}\left(2^{K-3}\right)+2$ & odd \\
3.2 & divide by 2 & 2 & $3^{2}\left(2^{K-4}\right)+1$ & $\ldots$ \\
$\ldots$ & $\ldots$ & $\ldots$ & $\ldots$ & even \\
$3(i-1)+1$ & $3 x+1$ rule & $i$ & $3^{i}\left(2^{K-2}(i-1)\right.$ & +4 \\
$3(i-1)+2$ & divide by 2 & $i$ & $3^{i}\left(2^{K-2 i+1}\right)+2$ & even \\
$3 i$ & divide by 2 & $i$ & $3^{i}\left(2^{K-2 i}\right)+1$ & odd \\
\hline
\end{tabular}

Table 1. A Collatz sequence for $2^{K}+1$.

For $K=2 i-1$, for some positive integer $i$ (i.e. for odd $K$ ), the Collatz sequence in Table 1 has $3^{i}\left(2^{K-2 i+1}\right)+2=3^{i}+2=3^{(K+1) / 2}+2$ at step $3(i-1)+2$; and for $K=2 i$, for a 
positive integer $i$ (i.e. for even $K$ ), it has $3^{i}\left(2^{K-2 i}\right)+1=3^{i}+1=3^{K / 2}+1$ at step $3 i$. In both cases ( $K$ odd or even), there exists a Collatz operation sequence for $2^{K}+1$ that yields an integer less than or equal to $3^{\lceil K / 2\rceil}+2$. Therefore, $2^{K}+1(K \geq 0)$ has a 1 -yielding Collatz sequence if $3^{\lceil K / 2\rceil}+2 \leq C$.

We note that for integer $K \geq 4,2^{K}+1>3^{\lceil K / 2\rceil}+2$. This means that although the Collatz conjecture is verified until $C$, the implication of Lemma 1 is that there exists an odd Collatz number of the form $2^{K}+1$ larger than $C$ for some $K$ where $3^{\lceil K / 2\rceil}+2 \leq C=5 \times 2^{60}$. Lemma 1 will remain to be true when the verified range (the integer $C$ ) for the Collatz conjecture increases in the future.

Lemma 2. For all integers $K \geq 0,(x)_{2} \geq 1$ such that $3^{\lceil K / 2\rceil}(x)_{2}+2 \leq C,\left(x 0^{K+2} 1\right)_{2}$ is an odd Collatz number.

Proof. This proof is similar to that of Lemma 1. For all $K \geq 0,\left(x 0^{K+2} 1\right)_{2}$ is a positive odd integer for which a Collatz sequence is described in Table 2. After step 0, steps are grouped by three as shown in Table 2.

\begin{tabular}{|l|l|c|l|l|}
\hline step $j$ & operation & group & $\begin{array}{l}\text { result } f_{j} \\
\text { (current integer in the sequence) }\end{array}$ & parity \\
\hline 0 & initialize & & $\left(x 0^{K+1} 1\right)_{2}=(x)_{2} 2^{K+3}+1$ & odd \\
1 & $3 x+1$ rule & 1 & $3(x)_{2} 2^{K+3}+4$ & even \\
2 & divide by 2 & 1 & $3(x)_{2} 2^{K+2}+2$ & even \\
3 & divide by 2 & 1 & $3(x)_{2} 2^{K+1}+1$ & odd \\
$3.1+1$ & $3 x+1$ rule & 2 & $3^{2}(x)_{2}\left(2^{K+1}\right)+4$ & even \\
$3.1+2$ & divide by 2 & 2 & $3^{2}(x)_{2}\left(2^{K}\right)+2$ & even \\
3.2 & divide by 2 & 2 & $3^{2}(x)_{2}\left(2^{K-1}\right)+1$ & odd \\
$\ldots$ & $\ldots$ & $\ldots$ & $\ldots$ & $\ldots$ \\
$3(i-1)+1$ & $3 x+1$ rule & $i$ & $3^{i}(x)_{2}\left(2^{K-2(i-1)}\right)+4$ & even \\
$3(i-1)+2$ & divide by 2 & $i$ & $3^{i}(x)_{2}\left(2^{K-2 i+1}\right)+2$ & even \\
$3 i$ & divide by 2 & $i$ & $3^{i}(x)_{2}\left(2^{K-2 i}\right)+1$ & odd \\
\hline
\end{tabular}

Table 2. A Collatz sequence for $\left(x 0^{K+2} 1\right)_{2}$.

For $K=2 i-1$, for some nonnegative integer $i$ (i.e. for odd $K$ ), the Collatz sequence in Table 2 has $3^{i}(x)_{2}\left(2^{K-2 i-1}\right)+2=3^{i}(x)_{2}+2=3^{(K+1) / 2}(x)_{2}+2$ at step $3(i-1)+2$; and for $K=2 i$, for a positive integer $i$ (i.e. for even $K$ ), it has $3^{i}(x)_{2}\left(2^{K-2 i}\right)+1=3^{i}(x)_{2}+1=3^{K / 2}(x)_{2}+1$ at step $3 i$. In both cases ( $K$ odd or even), a Collatz operation sequence for $\left(x 0^{K+2} 1\right)_{2}=(x)_{2} 2^{K+3}+1$ yields an integer less than or equal to $3^{\lceil K / 2\rceil}(x)_{2}+2$ included in the range $[1, C]$ where the conjecture has been verified by computers. Therefore, $(x)_{2} 2^{K+3}+1$ is a Collatz number for all $K \geq 0$ where $3^{\lceil K / 2\rceil}(x)_{2}+2 \leq C$. 
We note that for integers $K \geq 3,(x)_{2} \geq 1,\left(x 0^{K+2} 1\right)_{2}>3^{\lceil K / 2\rceil}(x)_{2}+2$. This means that there exists a Collatz number of the form $\left(x 0^{K+2} 1\right)_{2}$ larger than the computer-verified maximum $C$.

\section{Infinite sets of odd Collatz numbers}

In this section we show two infinite sets of odd Collatz numbers. Fig. 1 describes their elements.

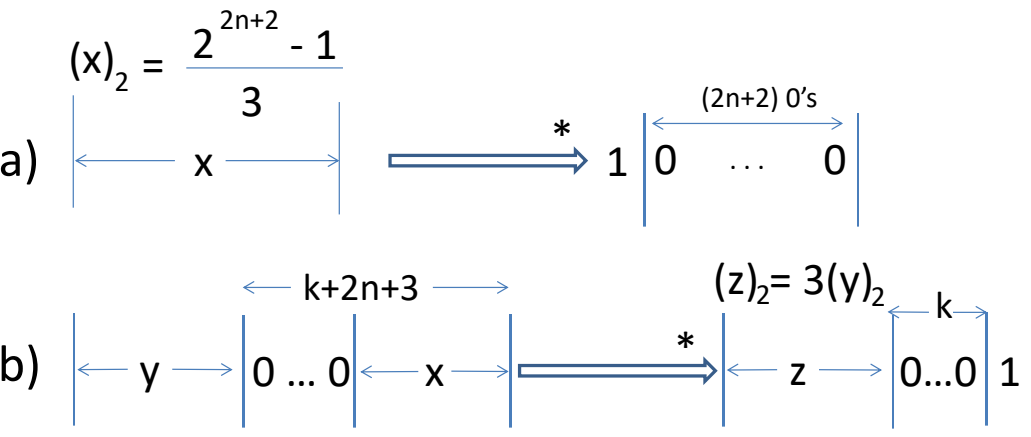

Figure 1. Two infinite sets of Collatz numbers. Each part describes a binary number and a number derived by applying the rules in Eq. (1): a) $\frac{2^{2 n+2}-1}{3}$ (an integer in set $\left.S_{1}\right) \Rightarrow^{*}\left(10^{2 n+2}\right)_{2}=2^{2 n+2}$; b) $(y)_{2} 2^{k+2 n+3}+(x)_{2}\left(\right.$ an integer in set $\left.S_{2}\right) \Rightarrow^{*}\left(z 0^{k} 1\right)_{2}$, where $(x)_{2} \in S_{1}, 3(x)_{2}+1=2^{2 n+2}$ for integer $n \geq 0 ; 3(y)_{2} 2^{k+1}+1=6(y)_{2} 2^{k}+1 \leq$ $C$ for integer $k \geq 0$. Both cases (a) and (b) eventually yield 1 .

Lemma 3. Let $S_{1}=\left\{2\left(\frac{2^{2 n+1}-2}{3}\right)+1=\frac{2^{2 n+2}-1}{3} \mid n\right.$ is a positive integer $\}$. Set $S_{1}$ is an infinite set of odd Collatz numbers.

Proof. We independently discovered a set of Collatz numbers (that we call $S_{1}$ in this paper) reported in Lemma 4.1 in [1]. However, our proof is different; and we focus on Collatz operation sequences. Fig. 1 (a) describes this case. First, we show that $S_{1}$ is a set of odd Collatz numbers. For every $n \geq 1$, we have the following implications

$$
\begin{aligned}
2^{2} \equiv 1(\bmod 3) & \Rightarrow 2^{2 n} \equiv 1(\bmod 3) \\
& \Rightarrow 2^{2 n+1} \equiv 2(\bmod 3) \\
& \Rightarrow 2^{2 n+1}-2 \equiv 0(\bmod 3)(\text { since }-2 \equiv 1(\bmod 3))
\end{aligned}
$$

Therefore, for every $n \geq 1,2\left(\frac{2^{2 n+1}-2}{3}\right)+1=\frac{2^{2 n+2}-1}{3}$ is a positive odd integer. For $2\left(\frac{2^{2 n+1}-2}{3}\right)+1$, a Collatz sequence is described in Table 3 .

As can be seen from this table, for every $n \geq 1,2\left(\frac{2^{2 n+1}-2}{3}\right)+1$ has a 1-yielding Collatz sequence having 1 at step $2 n+3$. In other words, the shortest 1-yielding Collatz operation sequence for $2\left(\frac{2^{2 n+1}-2}{3}\right)+1$ is $10^{2 n+2}$ (one application of $3 x+1$ rule, and then $2 n+2$ divisions by 2 ).

Clearly, $S_{1}$ contains infinitely many elements each of which is an odd number. This concludes the proof. 


\begin{tabular}{|l|l|l|}
\hline step $i$ & operation & result $f_{i}(y)$ \\
\hline 0 & initialize & $2\left(\frac{2^{2 n+1}-2}{3}\right)+1($ this is an odd number $)$ \\
\hline 1 & $3 f_{0}(y)+1$ & $\begin{array}{l}3\left(2\left(\frac{2^{2 n+1}-2}{3}\right)+1\right)+1=2^{2 n+2}-4+4 \\
=2^{2 n+2}(\text { a power of } 2)\end{array}$ \\
\hline$\ldots$ & $\ldots$ & $\ldots$ \\
\hline $2 n+3$ & $\frac{f_{2 n+2}(y)}{2}$ & 1 \\
\hline
\end{tabular}

Table 3. A Collatz sequence for $y=2\left(\frac{2^{2 n+1}-2}{3}\right)+1$.

Lemma 4. Let $S_{2}=\left\{(y)_{2} 2^{k+2 n+3}+(x)_{2} \mid(x)_{2} \in S_{1}\right.$, where $3(x)_{2}+1=2^{2 n+2}$ for some integer $n \geq 0 ; 6(y)_{2} 2^{k}+1 \leq C$ for some integer $k \geq 0$, and bit sequence $\left.y\right\}$. Set $S_{2}$ is an infinite set of odd Collatz numbers.

Proof. Fig. 1 (b) describes this case. Since $(x)_{2} \in S_{1}$, for some $n \geq 0,(x)_{2}=\frac{2^{2 n+2}-1}{3}$, and therefore, $3(x)_{2}+1=2^{2 n+2}$. The shortest 1-yielding Collatz operation sequence for $(x)_{2}$ is $10^{2 n+2}$. This sequence is a prefix of a 1-yielding Collatz operation sequence for $(y)_{2} 2^{k+2 n+3}+(x)_{2}$ for $k \geq 0$. The derivation from this sequence is summarized in Table 4 .

\begin{tabular}{|l|l|l|l|}
\hline step $i$ & operation & result $f_{i}\left((y)_{2} 2^{k+2 n+3}+(x)_{2}\right)$ & parity \\
\hline 0 & initialize & $(y)_{2} 2^{k+n+3}+(x)_{2}$ & odd \\
\hline 1 & multiply by $3 ;$ add 1 & $3(y)_{2} 2^{k+2 n+3}+3(x)_{2}+1=3(y)_{2} 2^{k+2 n+3}+2^{2 n+2}$ & even \\
\hline 2 & divide by 2 & $3(y)_{2} 2^{k+2 n+2}+2^{2 n+1}$ & even \\
\hline$\ldots$ & $\ldots$ & $\ldots$ & $\ldots$ \\
\hline $2 n+3$ & divide by 2 & $3(y)_{2} 2^{k+1}+1=6(y)_{2} 2^{k}+1 \leq C$ & odd \\
\hline$\ldots$ & $\ldots$ & $\ldots$ & $\ldots$ \\
\hline & & 1 & odd \\
\hline
\end{tabular}

Table 4. A Collatz sequence for $(y)_{2} 2^{k+2 n+3}+(x)_{2}$ for $(x)_{2} \in S_{1}$.

Since $k \geq 0,(y)_{2} 2^{k+2 n+3}+(x)_{2}$ is odd because $(x)_{2}$ is odd. The first operation will yield $3(y)_{2} 2^{k+2 n+3}+3(x)_{2}+1=3(y)_{2} 2^{k+2 n+3}+2^{2 n+2}$. After this step, the parity of the resulting number in each step $i$ is even until the result becomes $3(y)_{2} 2^{k+1}+1$ at step $i=2 n+3$ after $2 n+2$ divisions by 2 . It is given that $\left(3(y)_{2}\right) 2^{k+1}+1=6(y)_{2} 2^{k}+1 \leq C$. Therefore, $6(y)_{2} 2^{k}+1$ is guaranteed to have a 1-yielding Collatz sequence.

Clearly, set $S_{2}$ is infinite; and it contains only odd integers because the least significant bit positions in every element in $S_{2}$ are occupied only by some element in $S_{1}$ which is odd.

Theorem 1. For any given positive integer $B$, there exists:

1) at least one odd Collatz integer $\left(x_{1}\right)_{2}>B$ with the shortest 1-yielding Collatz operation sequence of the form $10^{2 n+2}$ for some $n \geq 0$; 
2) at least one odd Collatz integer $\left(x_{2}\right)_{2}>B$ with the shortest 1-yielding Collatz operation sequence of the form $10^{2 n+2} p$ where integer $n \geq 0$, and $p$ is the shortest 1-yielding Collatz operation sequence for some positive integer less than or equal to $C$.

Proof. To prove (1), we know from Lemma 3 that for any given integer $B$, there exists an odd integer $\left(x_{1}\right)_{2} \in S_{1}$ larger than $B$ such that $3\left(x_{1}\right)_{2}+1=2^{2 n+2}$, and $x_{1}$ has the shortest 1 -yielding Collatz operation sequence of the form $10^{2 n+2}$ for some integer $n \geq 0$.

To prove (2), take $\left(x_{1}\right)_{2}$ that satisfies case (1). That is, $\left(x_{1}\right)_{2} \in S_{1},\left(x_{1}\right)_{2}>B, 3\left(x_{1}\right)_{2}+1=$ $2^{2 n+2}$ for some $n \geq 0$, and $x_{1}$ has the shortest 1-yielding Collatz operation sequence of the form $10^{2 n+2}$. Consider using Lemma 4 to construct a new odd Collatz number $\left(x_{2}\right)_{2}$ from $\left(x_{1}\right)_{2}$ by setting $\left(x_{2}\right)_{2}=(y)_{2} 2^{k+2 n+3}+\left(x_{1}\right)_{2}$ for some integer $k \geq 0$. The integer $\left(x_{2}\right)_{2}$ has the shortest Collatz operation sequence with prefix $10^{2 n+2}$ with which $\left(x_{2}\right)_{2}=(y)_{2} 2^{k+2 n+3}+\left(x_{1}\right)_{2} \Rightarrow^{*}$ $6(y)_{2} 2^{k}+1$. Since $6(y)_{2} 2^{k}+1 \leq C$ there exists a 1-yielding Collatz operation sequence for $6(y)_{2} 2^{k}+1$. Let $p$ be the shortest of such sequences.

Therefore, $\left(x_{2}\right)_{2}$ has the shortest Collatz operation sequence $10^{2 n+2} p$. To obtain one such $p$, we can pick $y=10, k=0$. Applying the Collatz operation sequence $10^{2 n+2}$, we see that $(10)_{2} 2^{2 n+3}+\left(x_{1}\right)_{2} \Rightarrow^{*} 6(10)_{2}+1$, and $6(10)_{2}+1=(1101)_{2} \Rightarrow(101000)_{2} \Rightarrow(10100)_{2} \Rightarrow$ $(1010)_{2} \Rightarrow(101)_{2} \Rightarrow(10000)_{2} \Rightarrow(1000)_{2} \Rightarrow(100)_{2} \Rightarrow(10)_{2} \Rightarrow 1$.

Therefore, by noting the operations in this derivation, we see that $(10)_{2} 2^{2 n+3}+\left(x_{1}\right)_{2}$ has the shortest Collatz operation sequence $10^{2 n+2} 100010000$ which is of the form $10^{2 n+2} p$, where $p=100010000$ is the shortest Collatz operation sequence that yields 1 from $6(10)_{2}+1$.

\section{Conclusion}

We show that for any given positive integer $B$, we can construct odd Collatz numbers larger than $B$ with shortest 1-yielding Collatz operation sequences in more than one forms. Traditionally, computers are used to find ever-increasing range $[1, C]$ for verified Collatz numbers. In this paper, from the verified range $[1, C]$, first we construct finitely many odd Collatz numbers of the form $2^{K}+1>C$, and of the form $\left(x 0^{K+2} 1\right)_{2}$, where $x, K$ satisfy conditions we describe. Second, we present a method to construct an infinite set $S_{1}$ of odd Collatz numbers. Third, we develop a method to create another set $S_{2}$ by constructing, for every element $x$ in infinite set $S_{1}$, new odd Collatz numbers in $S_{2}$. Construction efforts of such sets can yield new insights into the Collatz conjecture, and large sets of integers can be shown to be Collatz numbers by construction without requiring computer verification. Clearly, the union of sets of Collatz numbers contains only Collatz numbers.

\section{References}

[1] Andrei, Ş, \& Masalagiu, C. (1997) About the Collatz conjecture, Acta Informatica, 35, $167-179$. 
[2] Maddux, C. D, \& Johnson, D. L. (1997) Logo: A Retrospective. New York: Haworth Press. p. 160.

[3] Lagarias, J. C. (1985) The $3 x+1$ problem and its generalizations. The American Mathematical Monthly 92(1), 3-23.

[4] Pickover, C. A. (2001) Wonders of Numbers. Oxford: Oxford University Press, pp. 116-118.

[5] Hofstadter, D. R. (1979). Gödel, Escher, Bach. New York: Basic Books, pp. 400-402.

[6] Guy, R. K. (2004) Unsolved problems in number theory (3rd ed.). Springer-Verlag, pp. 336-337.

[7] Guy, R. K. Don't try to solve these problems, Amer. Math. Monthly, 90 (1983), 35-41.

[8] Krasikov, I. (1989) How many numbers satisfy the $3 x+1$ conjecture?, Internat. J. Math. \& Math. Sci., 12(4), 791-796.

[9] Silva, T. O. e-S. Computational verification of the $3 x+1$ conjecture. Retrieved 27 November 2011. 